\title{
DOSES DE NITROGÊNIO NA PRODUÇÃO DE RABANETE FERTIRRIGADO E DETERMINAÇÃO DE CLOROFILA POR MEDIDOR PORTÁTIL NAS FOLHAS
}

\author{
Bárbara Rodrigues de Quadros; Edvar de Sousa da Silva; Luciana da Silva Borges; \\ Claudia de Araújo Moreira; Adriana Lima Moro; Roberto Lyra Villas Bôas. \\ Produção vegetal - Faculdade de Ciências Agronômicas, Universidade Estadual Paulista, \\ Botucatu, SP, barbaraunesp@yahoo.com.br
}

\section{RESUMO}

A determinação do teor relativo de clorofila por meio do clorofilômetro é utilizada para predizer a necessidade de adubação nitrogenada visando incrementar a produção em várias culturas. Este trabalho objetivou avaliar a resposta de doses de nitrogênio adicionadas ao solo via fertirrigação na produção de rabanete e a relação de clorofila com o teor de nitrogênio foliar da cultivar Redondo Vermelho. O delineamento experimental foram blocos casualizados, com cinco tratamentos $\left(0 ; 30 ; 60 ; 120 ; 240 \mathrm{~kg} \mathrm{~N} \mathrm{ha}^{-1}\right)$, cinco repetições, com dois vasos por parcela contendo quatro plantas em cada vaso, sendo duas úteis. Foram avaliados o diâmetro transversal da raiz, área foliar, índice de cor verde, teor de $\mathrm{N}$ foliar, a produção da parte aérea; número de raízes comerciais e não comerciais; massa de raízes total e comercial. As doses de $\mathrm{N}$ não interferiram na produção do rabanete e as leituras efetuadas com medidor portátil de clorofila não são muito precisas na verificação do nível de $\mathrm{N}$ no desenvolvimento da planta de rabanete.

UNITERMOS: Raphanus sativus, adubação nitrogenada, clorofilômetro.

QUADROS, B.R.; SILVA, E.S.; BORGES, L.S.; MOREIRA, C.A.; MORO, A.L.; VILLAS BÔAS, R.L. NITROGEN LEVELS ON FERTIGATED RADISH PRODUCTION AND CHLOROPHYLL DETERMINATION BY LEAVES PORTABLE METER

\section{ABSTRACT}

The relative chlorophyll determination is used to predict the need for nitrogen fertilization aiming to increase production in various cultures. The objective of this study was to evaluate the soil nitrogen dose response added to the soil via fertigation in radish production and the relation between chlorophyll and cultivar Redondo Vermelho leaf nitrogen content. Transverse diameter of root, leaf area, green index, leaf $\mathrm{N}$ contents, shoots (stem) production, number of commercial and noncommercial roots, and the total commercial mass roots were evaluated. The $\mathrm{N}$ doses didn't interfere in the radish production and the readings taken with portable chlorophyll meter are not very accurate in ascertaining the level of $\mathrm{N}$ on radish plant growth.

Keywords: Raphanus sativus, nitrogen fertilization, chlorophyll.

\section{INTRODUÇÃO}


O rabanete (Raphanus sativus L.) é uma brassicácea de porte reduzido, que nas cultivares de maior aceitação, produz raízes globulares, de coloração escarlate brilhante e polpa branca (Filgueira, 2005).

O rabanete, apesar de ser uma cultura de pequena importância em termos de área plantada, é cultivado em grande número de pequenas propriedades dos cinturões verdes das regiões metropolitanas. Uma das vantagens de se cultivar esta espécie é a possibilidade de obter renda durante o tempo transcorrido entre duas outras culturas de ciclo mais longo, pois além de ser relativamente rústica, apresenta ciclo muito curto (cerca de 30 dias), com retorno rápido (Cardoso \& Hiraki, 2001).

A fertirrigação, além de ser de grande utilidade para as plantas, pois o nutriente é fornecido juntamente com a água (essencial para sua absorção), apresenta ainda muitas outras vantagens, entre as quais a de melhor distribuição do fertilizante no campo e a possibilidade de maior parcelamento das adubações, aumentando a eficiência na utilização dos adubos pelas plantas (Duenhas et al., 2002).

Os nutrientes minerais podem afetar os níveis de alguns compostos orgânicos nas plantas devido à influência que exercem sobre os processos bioquímicos ou físiológicos, como a atividade fotossintética e a taxa de translocação de fotoassimilados (Ferreira et al., 2006).

O nitrogênio é o segundo nutriente mais exigido pelas hortaliças (Filgueira, 2005). Seu fornecimento via adubação, funciona como complementação à capacidade de suprimento dos solos, a partir da mineralização de matéria orgânica, geralmente baixos em relação às necessidades das plantas (Malavolta, 1990).

Em várias hortaliças, o nitrogênio desempenha papel fundamental no crescimento e no rendimento dos produtos colhidos (Oliveira et al.,2006).

Ganthi et al. (1989) e Singh et al. (1995) estudaram o efeito de doses de nitrogênio (0 a 100 $\mathrm{kg} / \mathrm{ha}$ ) em rabanete e relataram aumento de produção de folhas e raízes, assim como no número de raízes comerciais. Também Pell et al. (1990) observaram esta mesma tendência de aumento. Ressaltaram ainda que nas menores doses de nitrogênio a massa foliar representou uma maior fração da massa total da planta, em relação à massa de raízes, em comparação às maiores doses.

Embora o nitrogênio seja um importante nutriente para as hortaliças (Filgueira, 2005), pouco se conhece, ainda, a respeito das quantidades a serem utilizadas, que permitam a obtenção de rendimentos satisfatórios na cultura do rabanete.

A determinação do teor relativo de clorofila por meio do clorofilômetro está sendo utilizado para predizer a necessidade de adubação nitrogenada em várias culturas, dentre as principais: arroz (Balasubramanian et al., 2000); trigo (Bredemeier, 1999) e milho (Argenta et al., 2001). O teor de clorofila na folha é utilizado para predizer o nível nutricional de nitrogênio $(\mathrm{N})$ em plantas, devido ao fato da quantidade desse pigmento correlacionar-se positivamente com o teor de $\mathrm{N}$ na planta (Booij et al., 2000). Essa relação é atribuída, principalmente, ao fato de que 50 a $70 \%$ do $\mathrm{N}$ total das folhas ser integrante de enzimas (Chapman e Barreto, 1997) que estão associadas aos cloroplastos (Stocking e Ongun, 1962).

A análise química do tecido vegetal, normalmente utilizada para avaliar o estado nutricional da planta, pode ser demorada e de limitada utilização (Godoy, 2002). Em função disso, destaca-se a importância da praticidade e rapidez do uso do clorofilômetro. Entretanto, não há pesquisas que façam referências ao efeito do estado nutricional da cultura do rabanete através do medidor de clorofila.

O presente trabalho objetivou avaliar doses de $\mathrm{N}$ na produção de rabanete e a relação de clorofila com o teor de nitrogênio foliar. 


\section{MATERIAL E MÉTODOS}

O experimento foi conduzido em ambiente protegido, na Faculdade de Ciências Agronômicas da UNESP, Campus de Botucatu, estado de São Paulo com coordenadas geográficas de $21^{\circ} 26^{\prime}$ latitude sul, $48^{\circ} 26^{\prime}$ longitude oeste e altitude de $740 \mathrm{~m}$.

A estufa possuía $30 \mathrm{~m}$ de comprimento, $7 \mathrm{~m}$ de largura e $3 \mathrm{~m}$ de pé-direito, coberta com filme de polietileno transparente de baixa densidade com 150 micras de espessura.

$\mathrm{O}$ delineamento experimental utilizados foram blocos casualizados, com cinco tratamentos (doses de nitrogênio) e cinco repetições, com dois vasos por parcela contendo quatro plantas em cada vaso, sendo duas úteis.

O material colocado nos vasos foi retirado de um perfil de solo classificado como Latossolo Vermelho distrófico típico (Embrapa, 1997) com as seguintes características químicas (Raij et al., 2001): $\mathrm{pH}\left(\mathrm{CaCl}_{2}\right)$ de 4,1; 18,0 $\mathrm{g} \mathrm{dm}^{-3}$ de M.O.; 3,0 $\mathrm{mg} \mathrm{dm}^{-3}$ de P (resina); 0,2; 2,0 e 1,0 mmolc $\mathrm{dm}^{-3}$ de $\mathrm{K}^{+}, \mathrm{Ca}^{2+} \mathrm{e} \mathrm{Mg}^{2+}$, respectivamente; CTC de 75 mmolc $\mathrm{dm}^{-3}$ e saturação por bases de $4 \%$.

Com base na análise química do solo foi realizada a correção do solo e adubação de plantio. A calagem foi realizada e o solo permaneceu incubado por 30 dias antes da semeadura nos vasos, utilizando-se calcário dolomítico de alta reatividade (PRNT $=91 \%$ ), de modo a elevar-se a saturação por bases a $80 \%$, conforme recomendação sugerida por Trani \& Raij (1997).

A adubação de plantio correspondeu ao fornecimento de $\mathrm{N}$ na forma de uréia e de $\mathrm{K}_{2} \mathrm{O}$ na forma de cloreto de potássio. Todo o fósforo foi fornecido apenas no plantio na forma de superfosfato triplo $\left(42 \%\right.$ de $\mathrm{P}_{2} \mathrm{O}_{5}$ e $\left.13 \% \mathrm{Ca}\right)$ e termofosfato de yoorin máster $\left(17,5 \%\right.$ de $\mathrm{P}_{2} \mathrm{O}_{5}$, $0,10 \%$ de $\mathrm{B}$ e $0,55 \%$ de $\mathrm{Zn}$ ).

A adubação de cobertura foi realizada aos 14, 21 e 28 dias após a semeadura, de acordo com recomendação de Trani \& Raij et al. (1997), para a produção de rabanete, com o fornecimento de $\mathrm{N}$ e $\mathrm{K}$, na forma de uréia e cloreto de potássio, respectivamente a cada sete dias. Além disso, foi feita uma única aplicação de boro na forma de ácido bórico.

A semeadura foi realizada manualmente, utilizando três sementes por cova da cultivar Redondo Vermelho Gigante. Por ocasião do desbaste manteve-se duas plantas de $6 \mathrm{~cm}$.

A irrigação foi realizada conforme a exigência da cultura, através do monitoramento com tensiômetro. O manejo da irrigação foi realizado através de uma bateria de três tensiômetros de mercúrio instalados em cada tratamento, em parcelas tomadas aleatoriamente. A curva de retenção de água no solo, necessária para o manejo de irrigação via tensiometria, foi obtida segundo metodologia proposta por Richards (1965), determinando uma relação entre valores de umidade do solo e potencial matricial. Os dados para obtenção da curva foram obtidos no Laboratório de Relações Água - Solo do Departamento de Engenharia Rural da FCA/UNESP - Botucatu. As irrigações foram realizadas para elevar o potencial de água no solo de $-25 \mathrm{kPa}$ para $-10 \mathrm{kPa}$.

As doses de $\mathrm{N}$ utilizadas foram variadas em função da dose recomendada por Raij et al. (1997) para a produção de rabanete, que resultaram nos seguintes tratamentos: T0: tratamento sem nitrogênio (testemunha); T30: metade da dose mínima de nitrogênio recomendada $\rightarrow 30 \mathrm{~kg} \mathrm{ha}^{-1}$ de N; T60: dose mínima de nitrogênio $\rightarrow 60 \mathrm{~kg} \mathrm{ha}^{-1}$ de N ; T120: o dobro da dose mínima de nitrogênio $\rightarrow 120 \mathrm{~kg} \mathrm{ha}^{-1}$ de N; T240: quatro vezes a dose mínima de nitrogênio $\rightarrow 240 \mathrm{~kg} \mathrm{ha}^{-1}$ de $\mathrm{N}$. 
A colheita foi realizada aos 35 dias após a semeadura. Na colheita foram avaliados diâmetro transversal da raiz medido através de paquímetro digital em $\mathrm{mm}$; área foliar medida através do uso de um integrador de área foliar (LI-COR, LI 3000) $\mathrm{em} \mathrm{m}^{2}$; índice de cor verde medido em dois pontos da folha pré-madura de duas plantas por parcela através do aparelho clorofilômetro, que calcula os teores de clorofila com base na quantidade de luz transmitida pela folha em dois comprimentos de ondas, com diferentes absorbâncias da clorofila em spad; teor de $\mathrm{N}$ foliar em $\mathrm{g} \mathrm{Kg}^{-1}$ (30 dias após a semeadura), obtido através de digestão sulfúrica, conforme metodologia apresentada por Malavolta et al. (1997); a massa da parte aérea em g; número de raízes comerciais e não comerciais feita através de contagens; massa de raízes total e comercial realizada com auxílio de uma balança digital com três casas decimais de precisão em g. Considerou-se comercial a raiz com pelo menos dois $\mathrm{cm}$ de diâmetro que não apresentasse rachadura. Obteve-se, com estes resultados, as massas médias de raiz comercial e total em cada parcela.

Os resultados foram submetidos à análise de variância e em caso de efeito significativo para doses de nitrogênio, de acordo com o teste $\mathrm{F}$, foi realizada a análise de regressão para verificar o efeito de doses de nitrogênio nas características avaliadas. Realizou-se a análise de correlação de Pearson entre índice de cor verde e teor de $\mathrm{N}$ foliar.

\section{RESULTADOS E DISCUSSÃO}

De acordo com as análises de variâncias não foram obtidos efeitos significativos $(\mathrm{P}<0,05)$ das doses de $\mathrm{N}$ empregadas para as características de área foliar (média $\left.69,09 \mathrm{~cm}^{2}\right)$, número de folhas (média 21,16), diâmetro (média 2,35mm), número de raiz comercial (média 2,92), massa média de raiz comercial (média 170,31g), massa média de raiz total (média $180,25 \mathrm{~g}$ ) e índice de cor verde (Spad). No entanto, houve efeito significativo para o teor de nitrogênio foliar. A média da característica significativa avaliada se ajustou ao modelo linear de regressão, onde pela derivada da equação, calculou-se a dose de $\mathrm{N}$ responsável pela produtividade máxima estimada (Figura 1).

$\mathrm{O}$ teor de nitrogênio foliar aumentou de forma linear à medida que as doses de $\mathrm{N}$ foram aumentadas. $\mathrm{O}$ valor mais elevado para o teor de nitrogênio $\left(59 \mathrm{~g} \mathrm{Kg}^{-1}\right)$, no intervalo de estudo, foi obtido com a aplicação da dose máxima de N (240 kg ha-1) (Figura 1). A dose 240 $\mathrm{kg} \mathrm{ha}^{-1}$ está muito acima da recomendada por Raij et al. (1997) para a produção de rabanete $\left(60 \mathrm{~kg} \mathrm{ha}^{-1}\right)$, o que pode ter levado a planta a um estado de consumo de luxo, onde após atingir seu crescimento e produtividade máxima continuou absorvendo nitrogênio, refletindose de forma significativa somente no teor de nitrogênio foliar e não nas demais características avaliadas. Segundo Marschner (1995), esse fato é evidenciado pelo incremento de seu teor nos tecidos, embora essa maior quantidade não resulte em aumento de crescimento ou produtividade, uma vez que a planta já atingiu seu apogeu. 


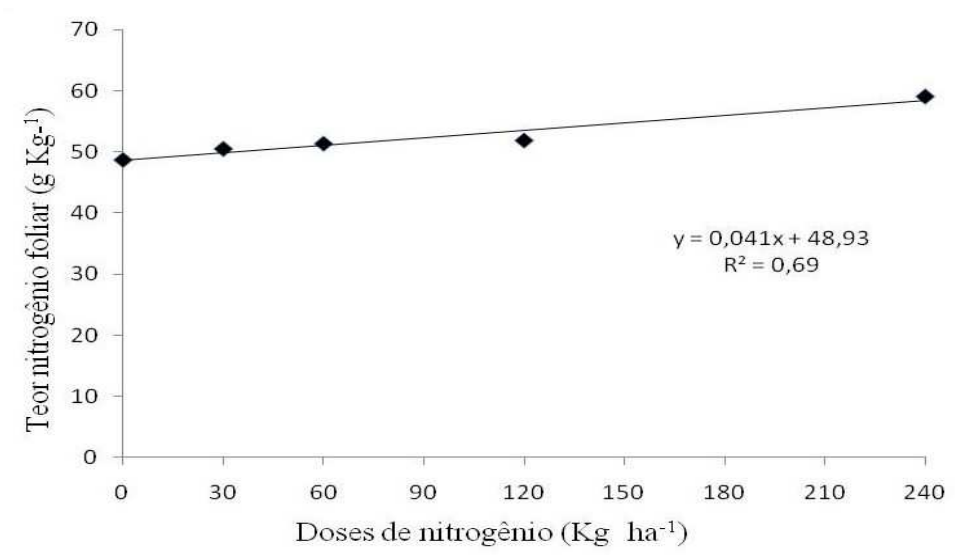

Figura 1. Teor de nitrogênio foliar em rabanete cv. Redondo vermelho gigante submetido a cinco doses de N. Botucatu, UNESP, 2009

A análise de correlação de Pearson evidenciou que as médias da leitura do clorofilômetro não se correlacionaram significativamente com o teor de nitrogênio $(-0,046 \mathrm{~ns})$. A falta de correlação entre leitura com clorofilômetro e teor de $\mathrm{N}$ na folha podem indicar que boa parte do $\mathrm{N}$ absorvido é provavelmente utilizado para a produção de outras estruturas na planta e não no acúmulo na folha para formação da clorofila, visto que a quantidade de nitrato presente deverá ser pequena, pois o mesmo é tóxico para a planta. Portanto, as leituras efetuadas com medidor portátil de clorofila com o objetivo de verificar o nível de $\mathrm{N}$ na planta, não são precisas no desenvolvimento do rabanete. Guimarães et al (1999) também não obtiveram correlações significativas com a concentração de N-org e N-total e concentrações de clorofila no limbo das folhas de plantas de tomate determinadas pelo método do medidor portátil SPAD-502 (CS). Argenta et al (2001) ao analisarem a relação entre leitura do clorofilômetro e teor de $\mathrm{N}$ na folha de dois híbridos de milho, constataram que, no estádio de seis a sete folhas, não houve relação entre as duas variáveis.

$\mathrm{O}$ teor de matéria orgânica no solo é considerado médio $\left(18,0 \mathrm{~g} / \mathrm{dm}^{3}\right)$. Esse teor pode ter disponibilizado nitrogênio para as plantas de rabanete, fazendo com que as mesmas não respondessem às doses de $\mathrm{N}$ aplicadas com relação à produtividade. Outro fator que pode ter influenciado é a eficiência de recuperação ou absorção do $\mathrm{N}$ aplicado por espécies olerícolas, que chega a ser igual ou até abaixo de $50 \%$, como observado por Cardoso \& Hiraki (2001) na cultura do rabanete, que ao aplicarem as doses de $\mathrm{N}(100 ; 200$ e $300 \mathrm{~kg} / \mathrm{ha}) \mathrm{na}$ forma de nitrato de cálcio aos 20 dias após a semeadura, constataram que não houve influencia das doses e a produção foi baixa.

\section{CONCLUSÃO}

As doses de $\mathrm{N}$ não interferiram na produção do rabanete.

As leituras efetuadas com medidor portátil de clorofila não são precisas na verificação do nível de $\mathrm{N}$ no desenvolvimento da planta de rabanete.

\section{REFERÊNCIAS BIBLIOGRÁFICAS}


ARGENTA, G. et al. Relação da leitura do clorofilômetro com os teores de clorofila extraível e de nitrogênio na folha de milho. Revista Brasileira de Fisiologia Vegetal, Lavras, v. 13, n. 2, p. 158-167, 2001.

BALASUBRAMANIAN, V. et al. Adaptation of the chlorophyll meter (SPAD) technology for real-time $\mathrm{N}$ management in rice: a review. International Rice Research Institute, Philippines, v. 5, p. 25-26, 2000.

BREDEMEIER, C. Predição da necessidade de nitrogênio em cobertura em trigo e aveia. 1999. 101p. Dissertação (Mestrado) - Universidade Federal do Rio Grande do Sul, Porto Alegre, 1999.

BOOIJ, R.; VALENZUELA, J.L.; AGUILERA, C. Determination of crop nitrogen status using non-invasive methods. In: HAVERKORT, A.J.; MACKERRON, D.K.L. (Ed.). Management of nitrogen and water in potato production, Wageningen Pers, 2000. p.7282.

CARDOSO, A.I.I.; HIRAKI, H. Avaliação de doses e épocas de aplicação de nitrato de cálcio em cobertura na cultura do rabanete. Horticultura Brasileira, Brasília, v. 19, n. 3, p. 196199, 2001.

CHAPMAN, S. C.; BARRETO, H. J. Using a chlorophyll meter to estimate specific leaf nitrogen of tropical maize during vegetative growth. Agronomy Journal, Madison, v. 89, n. 4, p. 557-562, 1997.

DUENHAS, L.H. et al. Fertirrigação com diferentes doses de N P K e seus efeitos sobre a produção e qualidade de frutos de laranja (Citrus sinensis O.) 'Valência'. Revista Brasileira de Fruticultura, Jaboticabal, v. 24, n.1, p. 214-218, 2002.

EMBRAPA. Centro Nacional de Pesquisa de Solos. Manual de métodos de análise de solos. 2 ed. rev. e atual. Rio de Janeiro: EMBRAPA, 1997. 212p.

FERREIRA M.M.M.; FERREIRA G.B.; FONTES P.C.R.; DANTAS J.P. Qualidade do tomate em função de doses de nitrogênio e da adubação orgânica em duas estações. Horticultura Brasileira, Brasília, v. 24, p. 141-145, 2006.

FILGUEIRA, F. A R. Novo Manual de olericultura: agrotecnologia moderna na produção e comercialização de hortaliças. Viçosa: UFV, 2005. 402 p. 
GHANTI, P.; SOUNDA, G.; GHATAK, S. Effect of levels of nitrogen and soil moisture regimes ongrowth and yield of radish. Environment and Ecology, v. 7, n. 4, p. 957-959, 1989.

GODOY, L. J. G. Manejo do nitrogênio em cobertura na cultura do milho (Zea mays L.) em solo arenoso baseado no índice relativo de clorofila. 2002. 94f. (Dissertação de Mestrado) - Faculdade de Ciências Agronômicas, Universidade Estadual Paulista "Júlio de Mesquita Filho", Botucatu, 2002.

GUIMARÃES, T.G. et al. Teores de clorofila determinados por medidor portátil e sua relação com formas de nitrogênio em folhas de tomateiro cultivados em dois tipos de solo. Bragantia, Campinas, v. 58, n.1, p. 209-216, 1999

MALAVOLTA, E.; VITTI, G.C.; OLIVEIRA, S.A. Avaliação do estado nutricional das plantas, princípios e aplicações. 2.ed. Piracicaba: Potafós, 1997. 319p.

MALAVOLTA E. Pesquisa com nitrogênio no Brasil-passado, presente e perspectivas. In: SIMPÓSIO BRASILEIRO SOBRE NITROGÊNIO EM PLANTAS, 1., 1990, Itaguaí, RJ. Anais...Itaguaí: Sociedade Brasileira de Fisiologia Vegetal, 1990. p.89-177.

MARSCHNER, H. Mineral nutrition of higher plants. London : Academic, 1995. 889p.

OLIVEIRA A. P. et al. Produção de raízes de batata-doce em função do uso de doses de N aplicadas no solo e via foliar. Horticultura Brasileira, Brasília, v. 24, p.279-282, 2006.

PELL, E.J.; WINNER, W.E.; MOONEY, H.A. Response of radish to multiple stresses. I. Physiological and growth responses to changes in ozone and nitrogen. New Phytologist, Lancaster, v. 115, n. 3, p. 439-446, 1990.

RAIJ, B. VAN.; CANTAREllA, H.; QUAGGIO, J. A.; FURLANI, A. M. C. Recomendações de adubação e calagem para o Estado de São Paulo. 2.ed. Campinas, Instituto Agronômico de Campinas: 1997. 285p.

RAIJ, B.VAN.; ANDRADE, J.C.; CANTARELLA, H.; QUAGGIO, J.A. Análise química para avaliação da fertilidade de solos tropicais. Campinas: Instituto Agronômico, 2001. $285 p$.

RICHARDS, L. A., Physical conditions of water in soil. In: C. A. BLACK, D. D. EVANS, J. L. WHITE, L. E. ENSMINGE and F. E. CLARK, ed. Methods of soil analysis - Physical 
and mineralogical properties, including statistics of measurements and sampling. Madison, ASASSSA, 1965. p.128-152.

SINGH, V.B.; KAR, P.L.; TATUNG, T. Effect of nitrogen and phosphorus on growth, yield and nutrient uptake of radish cv. Meghalaya selection. Advances in Horticulture and Forestry, India, v. 4, p. 127-132, 1995.

STOKING, C. R.; ONGUN, A. The intracellular distribution of some metallic elements in leaves. American Journal of Botany, California, v. 49, p. 284- 289, 1962.

TRANI P. E.; RAIJ, B. VAN. Composição química e diagnose foliar. Boletim técnico IAC, Campinas, n. 100, p. 164, 1997. 